\title{
COMPUTER SOLUTION TO THE 17-POINT ERDŐS-SZEKERES PROBLEM
}

\author{
GEORGE SZEKERES $^{1}$ and LINDSAY PETERS ${ }^{\varpi 2}$
}

\author{
In memory of Paul Erdös
}

(Received 25 April, 2006; revised 15 October, 2006)

\begin{abstract}
We describe a computer proof of the 17-point version of a conjecture originally made by Klein-Szekeres in 1932 (now commonly known as the "Happy End Problem") that a planar configuration of 17 points, no 3 points collinear, always contains a convex 6-subset. The proof makes use of a combinatorial model of planar configurations, expressed in terms of signature functions satisfying certain simple necessary conditions. The proof is more general than the original conjecture as the signature functions examined represent a larger set of configurations than those which are realisable. Three independent implementations of the computer proof have been developed, establishing that the result is readily reproducible.

2000 Mathematics subject classification: primary 52C10.

Keywords and phrases: Erdos-Szekeres problem, Ramsey theory, convex polygons and polyhedra, generalized convexity.
\end{abstract}

\section{Introduction}

One of the early interests of Paul Erdôs, one that had a strong influence on his later work in combinatorial geometry and Ramsey theory, was the following problem of Esther Klein-Szekeres: Is it true that for $k>1$

$\left(\boldsymbol{P}_{k}\right)$ Every planar set of $n>2^{k-2}$ points (in general position, no 3 points collinear) contains a subset of $k$ points which form a convex $k$-gon (denoted a convex $k$-subset).

\footnotetext{
${ }^{1}$ (deceased), School of Mathematics, University of NSW, Sydney NSW 2052, Australia.

${ }^{2}$ Pacific Knowledge Systems, Australian Technology Park, Eveleigh NSW 1430, Australia; e-mail: l.peters@pks.com.au.

(C) Australian Mathematical Society 2006, Serial-fee code 1446-1811/06
} 
$\left(Q_{k}\right)$ There exists a planar configuration of $2^{k-2}$ points which contains no convex $k$-subset.

For $k=2,3$ and 4 both statements are trivially true (for $k=4$ by a simple argument by the proposer herself) but already $k=5$ presents difficulties. When the problem was posed (in 1932) it was not even clear that for any $k>4$ there exists an $n=n(k)$ such that a set of $n$ points in the plane always contains a convex subset of $k$ points, but the question was soon settled in the affirmative by Erdôs and Szekeres [3].

Nowadays the proof of existence of such an $n$ presents no difficulties and is a simple exercise in Ramsey theory. The following argument is from van Lint and Wilson, [9, page 26]. Number the points of the configuration from 1 to $n$ in an arbitrary manner, and provide each triangle $a b c, a<b<c$, with a signature + or - according to the (anticlockwise or clockwise) orientation of the $a b c$. It is easily seen that a non-convex quadrilateral always contains triangles of both kinds. On the other hand by Ramsey, if $n$ is sufficiently large, the configuration contains a $k$-subset $K$ with all its triangles having the same signature. Hence all quadrilaterals of $K$ are convex and therefore $K$ itself is convex. If $n_{0}(k)+1$ denotes the smallest $n$ such that any planar configuration of size $n$ contains a convex $k$-subset, the conjectures $\left(P_{k}\right)$ and $\left(Q_{k}\right)$ together assert that $n_{0}(k)=2^{k-2}$.

At the time when the conjectures $\left(P_{k}\right)$ and $\left(Q_{k}\right)$ were proposed they seemed to be a hazardous extrapolation from a few trivial and not very convincing cases, but a few years later Makai and Turán verified that indeed 9 points always contain a convex pentagon. As far as we know this proof has never appeared in print but some of us who knew of its existence saw in it modest support for the belief that $\left(P_{k}\right)$ is true for all $k$. (See Morris and Soltan [7], Kalbfleisch et al. [5] and Bonnice [1] for proofs for 9 points.) Many years later Erdôs and Szekeres [4] produced an explicit example of $2^{k-2}$ points which contained no convex $k$-gon, thereby confirming the truth of $\left(Q_{k}\right)$ for all $k>1$. This construction showed at any rate that $n_{0}(k) \geq 2^{k-2}$. For recent results on the upper bound for $n_{0}(k)$ see Toth and Valtr [8].

The main result of the present paper is a computer proof of $n_{0}(6)=16$, that is, 17 points in the plane always contain a convex 6-subset, further strengthening the general validity of $\left(P_{k}\right)$.

\section{Combinatorial convexity}

To carry out the computer proof of $n_{0}(6)=16$ we shall need a combinatorial description of $n$-point configurations in the plane and a suitable combinatorial definition of convexity. Neither of these is unique and a judicious selection is vital for the success of the quite formidable computer search. As in Section 1, we shall make use of signatures of triangles, but the numbering of points upon which the signatures depend 
will not be arbitrary but restricted to specific choices for a given configuration. In fact the conceptual freedom afforded by an arbitrary numbering offers no advantages for the demands of computerisation.

Let $F_{n}$ be the set of configurations $P=\left\{p_{1}, \ldots, p_{n}\right\}$ of $n$ points in the $(x, y)$ coordinate plane. By rotating any given configuration we can assume that the $x$ axis is neither perpendicular nor parallel to any of the $\left(\begin{array}{l}n \\ 2\end{array}\right)$ edges of the configuration and so the points $p_{i}$ will have different $x$ coordinates. Indeed, we assume that the points $p_{1}$ are ordered in terms of increasing $x$-coordinate. Assuming that no three points are on a line, every ordered triple $\left(p_{i}, p_{j}, p_{k}\right), 1 \leq i<j<k \leq n$, is then oriented either clockwise or anticlockwise, imparting a signature $\sigma(i, j, k)=-$ or + to the triples according to their orientation.

More formally let $T_{n}$ be the set of ordered triples $(i, j, k)$ of $S_{n}=\{1,2, \ldots, n\}$ and $\Sigma_{n}$ the set of signature functions

$$
\sigma: T_{n} \rightarrow\{+,-\}
$$

on $T_{n}$. Then every $P \in F_{n}$ induces a signature function $\sigma \in \Sigma_{n}$ (strictly speaking an equivalence class of such functions, depending on the $x$ direction) and we regard $P$ as a geometrical realisation of this $\sigma$.

To define a convex $k$-gon we make use of "cups" and "caps" as defined by Chung and Graham [2]. After some experimentation we found them to be the most effective for the computer search ahead. An ordered set

$$
C_{\xi, i}=\left[a_{0}, a_{1}, \ldots, a_{i}\right], \quad 1 \leq a_{0}<a_{1}<\cdots<a_{i} \leq n, \xi=+ \text { or }-,
$$

will be called a $\xi$-chain of length $i \geq 1$ if $\sigma\left(a_{\mu-2}, a_{\mu-1}, a_{\mu}\right)=\xi$ for all $2 \leq \mu \leq i$. In particular, $\left[a_{0}, a_{1}\right]$ is a $\xi$-chain of length 1 for both $\xi=+$ and - . Here $C_{+, i}$ represents what Chung and Graham call an $(i+1)$-cup and $C_{-, i}$ an $(i+1)$-cap; we shall use either of these names, whichever is more convenient.

DEFINITION. A $k$-subset is called convex relative to $\sigma$ if it is the union of a cup-cap pair with common endpoints, that is, $C_{+, i} \cup C_{-, j}, C_{+, i}=\left[a_{0}, a_{1}, \ldots, a_{i}\right], C_{-, j}=$ $\left[b_{0}, b_{1}, \ldots, b_{j}\right]$ with total length $i+j=k, a=a_{0}=b_{0}, a_{i}=b_{j}=b$, and $C_{+, t} \cap C_{-, \jmath}=\{a, b\}$.

We will adopt this definition of convexity for $\sigma$, whether or not $\sigma$ is induced by some $P \in F_{n}$. Note that only triples formed by consecutive members of the chain were required to have the same signature, but if $\sigma$ is induced by a geometric configuration then of course all ordered triples of the chain have the same signature. We have chosen a weakest possible condition for $\xi$-chains so that they should represent Chung-Graham cups and caps whenever $\sigma$ is induced by some $P \in F_{n}$, where the points are ordered in terms of increasing $x$-coordinate. We can then ask whether the combinatorial version of $\left(P_{k}\right)$ is valid for arbitrary $\sigma$, that is, 
$\left(\boldsymbol{P}_{\sigma, k}\right)$ If $n>2^{k-2}$ then relative to any $\sigma \in \Sigma_{n}$ there is a convex $k$-subset of $S_{n}$.

As before, the existence of such an $n$ is established by the Ramsey argument of van Lint and Wilson. The conjecture $\left(P_{\sigma, k}\right)$ is considerably stronger than $\left(P_{k}\right)$ since only a small fraction of signature functions have a geometrical realisation. If $\sigma$ is induced by some $P \in F_{n}$ and $a b c d, 1 \leq a<b<c<d \leq n$, is a convex quadrilateral then writing

$$
\begin{array}{ll}
\sigma_{1}=\sigma(a, b, c), & \sigma_{2}=\sigma(a, b, d), \\
\sigma_{3}=\sigma(a, c, d), & \sigma_{4}=\sigma(b, c, d),
\end{array}
$$

we must have

$$
\sigma_{1}=\sigma_{2}, \quad \sigma_{3}=\sigma_{4}
$$

Similarly if $a b c d$ is a concave quadrilateral, that is, a triangle $a b d$ with $c$ inside the triangle, or a triangle $a c d$ with $b$ inside it, then we must have

$$
\sigma_{1}=-\sigma_{4}, \quad \sigma_{2}=\sigma_{3}
$$

These are easily verified by examining all possible 4-point configurations in the plane. Consequently the realisable signature functions are restricted to one of the (mutually exclusive) conditions (2.1) and (2.2) which must then be satisfied by all 4-subsets of $S_{n}$. This gives 8 possibilities (out of 16) for the signature values of any quadrilateral:

\begin{tabular}{rrrr}
$\sigma_{1}$ & $\sigma_{2}$ & $\sigma_{3}$ & $\sigma_{4}$ \\
\hline$\xi$ & $\xi$ & $\xi$ & $\xi$ \\
$\xi$ & $\xi$ & $\xi$ & $-\xi$ \\
$\xi$ & $\xi$ & $-\xi$ & $-\xi$ \\
$\xi$ & $-\xi$ & $-\xi$ & $-\xi$
\end{tabular}

where $\xi=+$ or - . We denote by $\Sigma_{n}^{*}$ the subset of those $\sigma \in \Sigma_{n}$ which satisfy one of the geometric constraints (2.1) or (2.2) for all 4-subsets of $S_{n}$.

Of course if $\left(P_{\sigma, k}\right)$ is true for all $\sigma \in \Sigma_{n}$ then it is also true for those special $\sigma$ for which all 4-subsets of $S_{n}$ belong to the 8 classes listed in (2.3). Therefore $\left(P_{\sigma, k}\right)$ implies the much weaker conjecture

$\left(P_{\sigma, k}^{*}\right)$ If $n>2^{k-2}$ then relative to any given $\sigma \in \Sigma_{n}^{*}$ there exists a convex $k$-subset.

Of course $\left(P_{k}\right)$ is an immediate consequence of $\left(P_{\sigma, k}^{*}\right)$ and it is natural to ask whether the two are actually equivalent. In other words, are the conditions (2.3) not only necessary but sufficient for $\sigma$ to be induced by some $P \in F_{n}$ ? The answer is no, and Knuth ([6, Figure 1, page 26]) shows an example of a 9-point non-realisable configuration satisfying (2.3). Hence $\left(P_{\sigma, k}^{*}\right)$ remains a stronger conjecture than $\left(P_{k}\right)$. 
At first sight, it looks fairly unlikely that $\left(P_{\sigma, k}\right)$ should be true. Yet $\left(P_{\sigma, 4}\right)$ is true for all $\sigma \in \Sigma_{5}$, that is, $S_{5}$ always contains a convex 4-subset. For suppose that all 4-subsets of $S_{5}$ are non-convex. Then assuming $\sigma(1,2,3)=+$, each of the following implications follows from the preceding one:

$$
(123)^{+} \rightarrow(235)^{-} \rightarrow(245)^{-} \rightarrow(124)^{+} \rightarrow(134)^{+} \rightarrow(345)^{-} \rightarrow(234)^{+} \rightarrow(123)^{-},
$$

where we have used the simplified notation $(i j k)^{\xi}$ to denote $\sigma(i, j, k)=\xi$.

For example, if $(235)^{+}$then $[1,2,3,5]$ is a 4-cup and the 4-subset 1235 is convex, contrary to assumption. Similarly in the second step if $(245)^{+}$then $[2,3,5]$ and $[2,4,5]$ are a 3-cup and 3-cap respectively and so the 4-subset 2345 is convex, again contrary to assumption. The other steps are variants of the first two. We end up with $(123)^{-}$, contradicting the original assumption. It follows that $S_{5}$ must contain a convex 4-subset.

This demonstration has the merit of being purely combinatorial (no reference to geometrical constraints), and it paves the way for a computer proof of $\left(P_{\sigma, 5}\right)$ in Section 3 and $\left(P_{\sigma, 6}^{*}\right)$ in Section 4. In particular, $\left(P_{\sigma, 5}\right)$ is true, a much stronger result than that of Makai and Turán. Its main interest is that, perhaps against expectations, $\left(P_{\sigma, k}\right)$ is true in the first non-trivial case $k=5$.

The proof of $\left(P_{\sigma, 6}^{*}\right)$ was much more time consuming than that of $\left(P_{\sigma, 5}\right)$, hardly surprising since it implies the validity of $\left(P_{6}\right)$, that is, the 17-point conjecture. It would be of great interest to also verify $\left(P_{\sigma, 6}\right)$, but at present this seems to be out of reach.

The algorithm described in Sections 3 and 4 has been implemented independently by each of the authors, and more recently, also by B. McKay.

\section{Computer proof of $\left(P_{\sigma, 5}\right)$}

THEOREM 1. For every $\sigma \in \Sigma_{9}$ there is a convex 5 -subset of $S_{9}$.

Take an arbitrary $\sigma \in \Sigma_{9}$ over $S_{9}=\{1,2, \ldots, 9\}$. Every such $\sigma$ is represented as a state of an array $A_{m}=\left\{a_{1}, \ldots, a_{m}\right\}$ of triples, of size $m=\left(\begin{array}{l}9 \\ 3\end{array}\right)=84$, and where each element $a_{t}$ takes the value + or - . That is, if $i$ corresponds to the triple $a b c$, then $a_{i}=\sigma(a, b, c)$. Here $A_{m}$ is introduced simply as a computational convenience, and in particular, during the search we will incrementally assign elements of $A_{m}$. A partially assigned $A_{m}$ therefore represents a subset of $\Sigma_{9}$.

The ordering of the elements of $A_{m}$ is not important, and so we choose

$$
a_{1}=\sigma(1,2,3), a_{2}=\sigma(1,2,4), a_{3}=\sigma(1,3,4), \ldots, a_{84}=\sigma(7,8,9) \text {. }
$$

As there are $2^{m}$ possible states of the array, a simple exhaustive search is of course not feasible and it is necessary to organise the search more efficiently. Whilst the 
following algorithm is not the simplest that can be used for the $n=9$ case, it is described here as it is able to be extended to deal with the $n=17$ case.

Let $a b c d e$ be any (ordered) set of five points of $S_{9}$. It forms a convex 5 -subset if and only if its $\left(\begin{array}{l}5 \\ 3\end{array}\right)=10$ triples satisfy one of the four relations (termed convex relations):

$$
\begin{array}{ll}
R_{1}: \sigma(a b c)=\sigma(b c d)=\sigma(c d e), & R_{2}: \sigma(a b c)=\sigma(b c e)=-\sigma(a d e), \\
R_{3}: \sigma(a b d)=\sigma(b d e)=-\sigma(a c e), & R_{4}: \sigma(a c d)=\sigma(c d e)=-\sigma(a b e) .
\end{array}
$$

Signatures that do not satisfy any of the relations (3.2) are termed concave.

The total number of convex relations on $S_{9}$ is therefore $4 \times\left(\begin{array}{l}9 \\ 5\end{array}\right)=504$. The hypothesis that for any 9 points there is at least one convex 5 -subset is therefore equivalent to the hypothesis that every (fully-assigned) state of the 84-element array $A_{m}$ must satisfy at least one of these 504 relations.

Consider now $\Sigma_{5}$ which is the set of signature functions on 5 points only. An arbitrary $\sigma \in \Sigma_{5}$ is represented as a state of $\left(\begin{array}{l}5 \\ 3\end{array}\right)=10$ elements, hence there are $2^{10}$ possible signature functions in $\Sigma_{5}$. A straightforward calculation will verify that 700 of these are convex. Denoting $\Omega \subset \Sigma_{5}$ as the subset of concave signature functions of $\Sigma_{5}$, we therefore have $|\Omega|=1024-700=324$.

Denote the signature functions in $\Omega$ by $\left\{\omega_{1}, \ldots, \omega_{324}\right\}$, where for fixed $i$, the 10 triples representing $\omega_{t}$ (in the same order as in (3.1)) are denoted $\left\{\omega_{t 1}, \ldots, \omega_{i 10}\right\}$. The ordering of the concave signature functions themselves is not important.

The hypothesis that every state of $A_{m}$ satisfies at least one of the 504 convex relations is therefore equivalent to the hypothesis that no state of $A_{m}$ can consist of signature functions wholly assigned from $\Omega$ for every 5 -subset in $S_{9}$.

Let $U_{5}=\left\{u_{1}, u_{2}, u_{3}, u_{4}, u_{5}\right\}$ denote the collection of contiguous 5-subsets in $S_{9}$ :

$$
u_{1}=[1,2,3,4,5], u_{2}=[2,3,4,5,6], \ldots, u_{5}=[5,6,7,8,9] .
$$

We say that a signature function (or more simply, a signature) in $\Omega$ is assigned to a 5 -subset in $U_{5}$ if it is assigned to the elements of $A_{m}$ corresponding to that 5-subset. That is, the restriction of $\sigma$ to $u$, is a translation of that signature. We note that if a signature in $\Omega$ is assigned to some $u_{j}, 1 \leq j \leq 4$, then the possible choices for assignment to $u_{j+1}$ are reduced as $u_{j}$ and $u_{j+1}$ share the 4 points $\{j+1, j+2$, $j+3, j+4\}$ and so share $\left(\begin{array}{l}4 \\ 3\end{array}\right)=4$ triples. Hence for each $\omega_{t} \in \Omega$ we define its compatible subset as the set of signatures $\omega_{l} \in \Omega$ such that

$$
\omega_{l 1}=\omega_{l 4}, \quad \omega_{l 2}=\omega_{i 7}, \quad \omega_{l 3}=\omega_{l 9}, \quad \omega_{l 4}=\omega_{i 10}
$$

A simple computer search verifies that each compatible subset contains no more than 27 signatures.

In brief, the algorithm performs an exhaustive search by successively assigning the signatures in $\Omega$ to the triples corresponding to the $u_{j}$ under the assumption that the 
hypothesis is false. That is, we assume that each state of $A_{m}$ is able to be assigned from signatures in $\Omega$, and show this leads to a contradiction in every case.

The algorithm relies on two key observations:

(1) The ordering of 5-subsets in (3.3) ensures that for a given signature, its compatible subset does not depend on $j$, that is, on whether that signature has been assigned to $u_{1}, u_{2}, u_{3}$ or $u_{4}$. Hence the compatible subsets for each signature in $\Omega$ need only be calculated once, prior to the search itself.

(2) A partially assigned state of $A_{m}$ can force further assignments, thereby eliminating large numbers of possible configurations from the search.

To illustrate the second observation, consider some partially assigned state of $A_{m}$, that is, not all elements are assigned + or - . We assume that the partially assigned state does not yet satisfy any of the convex relations, that is, if all three elements of $A_{m}$ corresponding to some convex relation in (3.2) are assigned, then that relation is not satisfied. We now note that assigning one more element of $A_{m}$ may necessarily determine the values of some other elements due to the assumption that none of the convex relations (3.2) are satisfied. For example, if $\sigma(a b c)=\sigma(b c d)=+$ then $\sigma(c d e)$ must necessarily take the value - to avoid the first relation in (3.2). These other assigned elements may, in turn, determine the values of further elements, and so on recursively.

In this way, the assignment of a signature from $\Omega$ to the elements of $A_{m}$ corresponding to some $u_{j}$ may cause further elements of $A_{m}$ to be necessarily assigned, further restricting the choices of compatible signatures to $u_{m}$ for $m>j$, and hence reducing the number of subsequent configurations required to be examined.

Furthermore, an element of $A_{m}$ necessarily assigned by considering one convex relation may cause some other convex relation to be satisfied, eliminating from the search all configurations represented by this partial assignment to $A_{m}$.

In this way, contradictions are generated with relatively few assignments needing to be made from $\Omega$. In fact, the computer search indicates that the only 5-subsets that ever need to be assigned are (in order) $u_{1}, u_{2}$, and $u_{3}$ (the forced assignments involve points 8 and 9).

The details of the algorithm are now described. It will terminate when a fully assigned state is found with no contradiction, or alternatively, when all possible configurations have led to a contradiction.

Algorithm 1. Step 1. Suppose we have a partial assignment of $A_{m}$ obtained by an assignment to $u_{1}, \ldots, u_{j}$ for some $j \leq 5$ by elements of $\Omega$. (Initially we simply assign the first element of $\Omega$ to $u_{1}$ for some fixed ordering of elements of $\Omega$ ). Check convex relations (3.2) to see if a contradiction has been reached or if $\sigma(i j k)$ for some unassigned triple $(i, j, k)$ is forced to avoid satisfying one of the convex relations. Repeat this step (that is, re-checking all the convex relations when a new 
element is necessarily assigned) until no more elements are necessarily assigned, or a contradiction is reached.

Step 2. If there is no contradiction found in the previous step, we have shown that the current assignments to $u_{1}, \ldots, u_{j}$ do not cause any of the convex relations to be satisfied. Check whether $A_{m}$ is now completely assigned. If it is, we have found a state where no convex relation is satisfied, and the search terminates with a counterexample found to Theorem 1 . If it isn't completely assigned, move to the next configuration to be checked as follows. If $\omega$ is currently assigned to $u_{j}$, assign the first possible element of the compatible subset for $\omega$ to $u_{j+1}$ and go to Step 1. We note that there could be triples in $u_{j+1}$ that have already been necessarily assigned, restricting the choices from the compatible subset for $\omega$ as mentioned above. If there are no remaining choices for assignments to $u_{j+1}$, we have derived a contradiction by the current assignments to $u_{1}, \ldots, u$, and so we go to Step 3 .

Step 3. If there was a contradiction found in Steps 1 or 2 we have shown that irrespective of the possible assignments of the remaining unassigned elements of $A_{m}$, the current assignments to $u_{1}, \ldots, u_{j}$ will necessarily cause one of the convex relations to be satisfied. Move to the next configuration to be checked as follows:

Step 4. Restore the state of $A_{m}$ to what it was prior to $u_{j}$ having its current assignment. Assign the next possible element to $u_{j}$, that is, the next possible element of the compatible subset of the element assigned to $u_{j-1}$ if $j>1$, or the next element of $\Omega$ if $j=1$, and go to Step 1 . If there are no remaining choices for assignments to $u_{j}$ we have derived a contradiction by the current assignments to $u_{1}, \ldots, u_{j-1}$ and so if $j>1$ we decrement $j$ and repeat this step. If $j=1$ and there are no remaining choices from $\Omega$ to $u_{1}$ the search terminates with all possible states having led to a contradiction.

\section{Some notes on efficiency}

- In Step 1, when some new element is assigned, only the convex relations involving the new element need to be checked, and not the complete list of 504 relations. Hence for each element $a_{l}$ of $A_{m}$, the set of relations involving $a_{l}$ are stored prior to the commencement of the search.

- We can take advantage of the linearity of the convex relations by only assigning half of the possible elements of $\Omega$ to $u_{1}$, for example, those elements for which $a_{1}=+$.

Using a $1.5 \mathrm{GHz}$ workstation, the search terminates at Step 4 in less than one second, establishing Theorem 1.

\section{Computer proof of $\left(P_{\sigma, 6}^{*}\right)$}

THEOREM 2. For every $\sigma \in \Sigma_{17}^{*}$ there is a convex 6-subset of $S_{17}$. 
The description of the 17-point proof will focus on the required extensions to the proof given above for the 9-point case. Unless indicated otherwise, the various entities ( $A_{m}, \Omega$ and so on) will be denoted by the same symbols as their 9-point counterparts.

The number of elements $m$ in $A_{m}$ required to represent a signature in $\Sigma_{17}$ over $S_{17}$ is now $\left(\begin{array}{c}17 \\ 3\end{array}\right)=680$. Let abcdef be any (ordered) set of six points of $S_{17}$. They form a convex 6-subset if and only if its $\left(\begin{array}{l}6 \\ 3\end{array}\right)=20$ triples satisfy one of the eight convex relations:

$$
\begin{aligned}
& R_{1}: \sigma(a b c)=\sigma(b c d)=\sigma(c d e)=\sigma(\text { def }), \\
& R_{2}: \sigma(a b c)=\sigma(b c d)=\sigma(c d f)=-\sigma(a e f), \\
& R_{3}: \sigma(a b c)=\sigma(b c e)=\sigma(c e f)=-\sigma(a d f), \\
& R_{4}: \sigma(a b d)=\sigma(b d e)=\sigma(d e f)=-\sigma(a c f), \\
& R_{5}: \sigma(a c d)=\sigma(c d e)=\sigma(d e f)=-\sigma(a b f), \\
& R_{6}: \sigma(a b c)=\sigma(b c f)=-\sigma(a d e)=-\sigma(d e f), \\
& R_{7}: \sigma(a b d)=\sigma(b d f)=-\sigma(a c e)=-\sigma(c e f), \\
& R_{8}: \sigma(a c d)=\sigma(c d f)=-\sigma(a b e)=-\sigma(b e f) .
\end{aligned}
$$

The total number of convex relations on $S_{17}$ is therefore $8 \times\left(\begin{array}{c}17 \\ 6\end{array}\right)=99,008$. A straightforward calculation verifies that $|\Omega|=184,556$ where $\Omega$ is now the set of concave signatures on $S_{6}$.

Let $U_{12}=\left\{u_{1}, u_{2}, \ldots, u_{12}\right\}$ denote the contiguous 6-subsets in $S_{17}$ :

$$
u_{1}=[1,2, \ldots, 6], u_{2}=[2,3, \ldots, 7], \ldots, u_{12}=[12,13, \ldots, 17] \text {. }
$$

Ideally we would attempt to assign all signatures from $\Omega$ to the 6-subsets in order to establish $\left(P_{\sigma, 6}\right)$. To date however, this has not been achieved due to the large number of signatures and consequent time required. However, by restricting the concave signatures to those satisfying the geometric conditions, the search is readily performed. We therefore define $\Omega^{*} \subset \Omega$ to be those concave signatures which satisfy relations in (2.3) for all 4-subsets in $S_{6}$.

As before, we note that if a signature in $\Omega^{*}$ is assigned to the elements of $A_{m}$ corresponding to a 6 -subset $u_{j}$, then the possible choices for assignment to $u_{j+1}$ are reduced as $u_{j}$ and $u_{j+1}$ share the 5 points $\{j+1, \ldots, j+5\}$ and so share $\left(\begin{array}{l}5 \\ 3\end{array}\right)=10$ triples. A simple computer search verifies that $\left|\Omega^{*}\right|=892$ and that for each signature in $\Omega^{*}$, its compatible subset contains no more than 18 signatures.

Two of the steps described in Algorithm 1 now need to be extended, as follows.

Extension to Algorithm 1, Step 1 In the 9-point case, we checked the convex relations to see if any other elements of $A_{m}$ are necessarily assigned, or if a contradiction is reached. Now however, we not only check the convex relations (4.1) but also 
the geometric conditions (2.3). If all four triples corresponding to a 4-subset of $S_{17}$ are assigned, but neither of the geometric conditions (2.1) or (2.2) is satisfied, then a contradiction has been reached. If two or three of the triples corresponding to a quadrilateral are assigned, then it could be that one or two of the remaining triples must necessarily be assigned to avoid a contradiction. For example, suppose $\sigma_{1}$ and $\sigma_{4}$ are both assigned + , with $\sigma_{2}$ and $\sigma_{3}$ unassigned. In this case, the only way to satisfy one of the geometric conditions (2.1) and (2.2) is if $\sigma_{2}$ and $\sigma_{3}$ are both assigned + .

Extension to Algorithm 1, Step 2 In the 9-point case, $j$ never exceeded 3, which is why the definition (3.3) sufficed. We now find however that $j$ will reach its maximum value of 12 . That is, there are combinations of signatures assigned to $u_{1}, \ldots, u_{12}$ which do not generate any contradictions. In this situation, we perform up to three more types of checks:

The $U_{13}$ check: We extend the $U_{12}$ to $U_{13}$ by including another 6-subset

$$
u_{13}=[9,13,14,15,16,17] .
$$

We have arbitrarily chosen point 9 , but any point from 1 to 11 would have sufficed. We now check all signatures to $u_{13}$ compatible with the one assigned to $u_{12}$ in the sense that they must agree on the 10 triples they have in common. As the triples shared between $u_{12}$ and $u_{13}$ are different to those shared between $u_{j}$ and $u_{j+1}, 1 \leq j \leq 11$, a second compatible subset is pre-calculated for each signature in $\Omega^{*}$, in readiness for assignments to $u_{13}$. We could extend these selected 6-subsets still further, but this was not found to be necessary in practice. If an assignment is made to $u_{13}$ that still does not lead to a contradiction, we go to the next step.

The one-bit check: We pick the first unassigned element $a_{l}$ of $A_{m}$ and arbitrarily assign it to + . We then perform the checks in Algorithm 1, Step 1 as described above (that is, both the convex and geometric checks). If a contradiction is generated, we restore the state of $A_{m}$ to what it was before assigning $a_{l}$ and now check the - assignment. If a contradiction is also generated we know that the current assignments to $u_{1}, \ldots, u_{13}$ necessarily lead to a contradiction, and we can proceed to Algorithm 1, Step 3. If either assignment to $a_{l}$ did not lead to a contradiction, we restore the state of $A_{m}$ to what it was before that assignment, and pick the next unassigned element of $A_{m}$. If we exhaust all unassigned elements of $A_{m}$ without generating a contradiction we proceed to the next step.

The two-bit check: This is similar to the one-bit check except that instead of picking just one unassigned element of $A_{m}$, we pick the first pair of unassigned elements. There are now four assignments to check $(++,+-,-+,--)$, and if all lead to a contradiction, we can again proceed to Algorithm 1, Step 3. If any of the four assignments do not lead to a contradiction, we pick another pair of unassigned elements, and so on. It was found that the two-bit check always succeeded in generating 
a contradiction at this point in the search.

Whilst the two-bit check by itself would generate a contradiction to any combination of signatures that the one-bit check had generated a contradiction for (and so subsumes the one-bit check), the number of pairwise checks means that this step is much slower to execute and so it is more efficient to do the one-bit check first.

The number of partial signatures that survived the $U_{13}$ check was $20,312,212$. The number that survived the one bit check was 23,339 .

There is evidently a certain arbitrariness in the three strategies above, and various combinations of these strategies or others will suffice to check those few combinations of signatures that do not lead to a contradiction when assigned to $u_{1}, \ldots, u_{12}$. For example, one of the authors (Szekeres) used a three-bit check instead of the $U_{13}$ check for those signatures that had slipped through the one- and two-bit checks. (In his implementation, B. McKay used a more efficient variation of the one-bit check in place of the two-bit check).

The algorithm described above is amenable to parallel execution, as each assignment of a signature from $\Omega^{*}$ to $u_{1}$ can be done independently of any other assignment to $u_{1}$. For example, one could have up to $\left|\Omega^{*}\right| / 2=446$ independent processes, each evaluating one of the possible assignments to $u_{1}$. Using modest computing platforms (less than $2 \mathrm{GHz}$ workstations) the time required to generate a contradiction from just one assignment to $u_{1}$ took between one hour and twenty hours, depending on the particular assignment. The total computing time to establish contradictions for all 446 assignments to $u_{1}$, and thus establish Theorem 2, was approximately $3,000 \mathrm{GHz}$ hours, that is, 1,500 hours of computing time using processors of up to $2 \mathrm{GHz}$.

\section{Proof of $\left(Q_{\sigma, k}^{*}\right)$}

In this section we shall prove the corresponding combinatorial (and weaker) version of $\left(Q_{k}\right)$ :

$\left(Q_{\sigma, k}^{*}\right.$ ) If $n=2^{k-2}$ there exists a $\sigma \in \Sigma_{n}^{*}$ which contains no convex $k$-subset.

We present this partly for completeness, and partly to show how the new combinatorial setting simplifies the original construction [4] if we don't insist on realisability.

LEMMA 1. Given $i+j=m-2 \geq 2$, there exists a $\sigma \in \Sigma_{\left(\begin{array}{c}m-2 \\ i-1\end{array}\right)}^{*}$ over $\left\{1, \ldots,\left(\begin{array}{c}m-2 \\ i-1\end{array}\right)\right\}$ which admits neither a $C_{+, i}$ of length $i$ nor a $C_{-, j}$ of length $j$.

The statement is trivially true (empty) when $i=1$ or $j=1$, hence we may assume

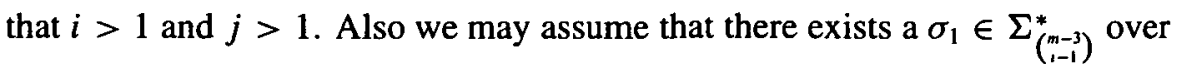

$$
\left\{1, \ldots,\left(\begin{array}{c}
m-3 \\
i-1
\end{array}\right)\right\}
$$


which admits neither a $C_{+, i}$ nor a $C_{-, j-1}$ and a $\sigma_{2} \in \Sigma_{\left(\begin{array}{c}m-3 \\ j-1\end{array}\right)}^{*}$ over

$$
\left\{\left(\begin{array}{c}
m-3 \\
i-1
\end{array}\right)+1, \ldots,\left(\begin{array}{c}
m-3 \\
i-1
\end{array}\right)+\left(\begin{array}{c}
m-3 \\
j-1
\end{array}\right)=\left(\begin{array}{c}
m-2 \\
i-1
\end{array}\right)\right\}
$$

which admits neither a $C_{+, i-1}$ nor a $C_{-, j}$. Extend these two signature functions to the whole set $\left\{1, \ldots,\left(\begin{array}{c}m-2 \\ t-1\end{array}\right)\right\}$ by setting

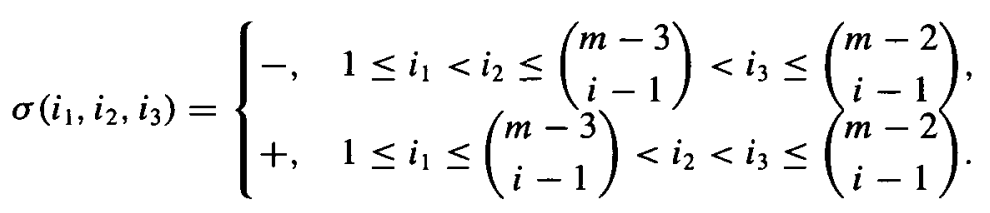

Now suppose there is a $C_{+, l}=\left[k_{0}, \ldots, k_{i}\right]$, then we must have $k_{0}<k_{1} \leq\left(\begin{array}{c}m-3 \\ i-1\end{array}\right)$ and $k_{i}>\left(\begin{array}{c}m-3 \\ i-1\end{array}\right)$ since $\sigma_{2}$ does not admit a $C_{+, i-1}$. Let $\mu \geq 1$ be such that

$$
1 \leq k_{\mu-1}<k_{\mu} \leq\left(\begin{array}{c}
m-3 \\
i-1
\end{array}\right)<k_{\mu+1} \leq\left(\begin{array}{c}
m-2 \\
i-1
\end{array}\right),
$$

then $\sigma\left(k_{\mu-1}, k_{\mu}, k_{\mu+1}\right)=-$ by definition, contrary to the assumption that $C_{+, i}$ is a cup. Similarly, suppose there is a $C_{-, J}=\left[k_{0}, \ldots, k_{j}\right]$ then we must have

$$
\left(\begin{array}{c}
m-3 \\
i-1
\end{array}\right)<k_{j-1}<k_{j} \leq\left(\begin{array}{c}
m-2 \\
i-1
\end{array}\right)
$$

and $\sigma\left(k_{v-1}, k_{v}, k_{v+1}\right)=+$ for suitable $\nu>\left(\begin{array}{c}m-3 \\ i-1\end{array}\right)$, by definition, again a contradiction.

The extended signature function satisfies the constraints (2.3). For instance, if $1 \leq i_{1}<i_{2}<i_{3} \leq\left(\begin{array}{c}m-3 \\ 1-1\end{array}\right)<i_{4}$ then $\sigma\left(i_{1}, i_{2}, i_{4}\right)=\sigma\left(i_{1}, i_{3}, i_{4}\right)=\sigma\left(i_{2}, i_{3}, i_{4}\right)=-$ and (2.3) is satisfied irrespective of the value of $\sigma\left(i_{1}, i_{2}, i_{3}\right)$. Similarly for the other two cases, hence $\sigma \in \Sigma_{\left(\begin{array}{c}m-3 \\ i-1\end{array}\right)}^{*}$.

To construct $\sigma \in \Sigma_{2^{k-2}}^{*}$ which does not admit a convex $k$-subset we proceed as follows: Let $p_{r}=\sum_{i=0}^{r}\left(\begin{array}{c}k-2 \\ i\end{array}\right)$ for $0 \leq r \leq k-2$ so that $p_{0}=1, p_{k-2}=2^{k-2}$. Let $\sigma_{j} \in \Sigma_{\left(\begin{array}{c}k-2 \\ j\end{array}\right)}^{*}$ over $P_{j}=\left\{p_{j-1}+1, \ldots, p_{j-1}+\left(\begin{array}{c}k-2 \\ j\end{array}\right)=p_{j}\right\}, 0<j<k-2$, be such that according to Lemma 1 it admits neither a $C_{+, j+1}$ nor a $C_{-, k-j-1}$. Setting $P_{0}=\{1\}$, $P_{k-2}=\left\{2^{k-2}\right\}$, then $\bigcup_{j=0}^{k-2}$ is a partition of $S=\left\{1,2, \ldots, 2^{k-2}\right\}$.

Define $\pi: S \rightarrow\{0,1, \ldots, k-2\}$ to be the projection $\pi(r)=i$ if $r \in P_{i}$. We can now extend the signature $\sigma_{j}$ to the whole of $S$ by setting

$$
\sigma\left(k_{1}, k_{2}, k_{3}\right)= \begin{cases}+, & \text { if } \pi\left(k_{1}\right) \leq \pi\left(k_{2}\right)<\pi\left(k_{3}\right), \\ -, & \text { if } \pi\left(k_{1}\right)<\pi\left(k_{2}\right)=\pi\left(k_{3}\right), \\ \sigma_{j}\left(k_{1}, k_{2}, k_{3}\right), & \text { if } \pi\left(k_{1}\right)=\pi\left(k_{2}\right)=\pi\left(k_{3}\right)=j .\end{cases}
$$


We show that this $\sigma$ does not admit a convex $k$-subset, that is, a pair of chains $C_{+, r}$, $C_{-, s}$ with common endpoints and $r+s=k$. For suppose $C_{+, r}=\left[a, a_{1}, \ldots, a_{r-1}, b\right]$ and $C_{-, s}=\left[a, b_{1}, \ldots, a_{s-1}, b\right]$ with $\pi(a)=i, \pi(b)=j, i \leq j$. If $i=j$ then by construction $r+s \leq k-2<k$. If $i<j$, then $\pi\left(b_{\mu}\right)=j$ for all $0<\mu<s$ and

$$
i=\pi(a)=\pi\left(a_{1}\right)=\cdots=\pi\left(a_{v}\right)<\pi\left(a_{v+1}\right)<\cdots<\pi\left(a_{r-1}\right)<j
$$

for some $v \leq i$. Hence $r \leq i+(j-i)=j, s \leq k-j-1, r+s \leq k-1<k$.

Next we verify that the conditions (2.3) hold for $\sigma$. Take any 4-subset $\left\{k_{1}, k_{2}, k_{3}, k_{4}\right\}$, $\pi_{\nu}=\pi\left(k_{v}\right), \nu=1, \ldots, 4$, so that $\pi_{1} \leq \pi_{2} \leq \pi_{3} \leq \pi_{4}$. We may assume $\pi_{1}<\pi_{4}$, otherwise the points $k_{v}$ would all be in the same $P_{i}$ and by construction would satisfy (2.3). Suppose that $\pi_{1} \leq \pi_{2} \leq \pi_{3}<\pi_{4}$. Then

$$
\sigma\left(k_{1}, k_{2}, k_{4}\right)=\sigma\left(k_{1}, k_{3}, k_{4}\right)=\sigma\left(k_{2}, k_{3}, k_{4}\right)=+
$$

by definition, and (2.3) is satisfied. Similarly if $\pi_{1} \leq \pi_{2}<\pi_{3}=\pi_{4}$ then

$$
\sigma\left(k_{1}, k_{2}, k_{3}\right)=\sigma\left(k_{1}, k_{2}, k_{4}\right)=+, \quad \sigma\left(k_{1}, k_{3}, k_{4}\right)=\sigma\left(k_{2}, k_{3}, k_{4}\right)=-.
$$

Finally if $\pi_{1}<\pi_{2}=\pi_{3}=\pi_{4}$ then $\sigma\left(k_{1}, k_{2}, k_{3}\right)=\sigma\left(k_{1}, k_{2}, k_{4}\right)=\sigma\left(k_{1}, k_{3}, k_{4}\right)=-$, and in all cases (2.3) is satisfied. Thus $\sigma \in \Sigma_{2^{t-2}}^{*}$.

\section{Acknowledgements}

The authors gratefully acknowledge the assistance of B. McKay in independently implementing and verifying the algorithm presented in Section 4 , and for his assistance in the preparation of this article.

\section{References}

[1] W. E. Bonnice, “On convex polygons determined by a finite planar set", Amer. Math. Monthly 81 (1974) 749-752.

[2] F. R. K. Chung and R. L. Graham, "Forced convex n-gons in the plane", Discrete Comput. Geom. 19 (1998) 367-371, Special Issue.

[3] P. Erdôs and G. Szekeres, "A combinatorial problem in geometry", Compositio Math. 2 (1935) $463-470$.

[4] P. Erdős and G. Szekeres, "On some extremum problems in elementary geometry", Ann. Univ. Sci. Budapest. Eötvös Sect. Math. 3-4 (1961) 53-63.

[5] J. D. Kalbfleisch, J. G. Kalbfleisch and R. G. Stanton, "A combinatorial problem on convex regions", in 1970 Proc. Louisiana Conf. on Combinatorics, Graph Theory and Computing, (Louisiana State Univ., Baton Rouge, 1970) 180-188.

[6] D. E. Knuth, Axioms and Hulls (Springer, Heidelberg, 1992). 
[7] W. Morris and V. Soltan, "The Erdốs-Szekeres problem on points in convex position. A survey", Bull. Amer. Math. Soc. (N.S.) 37 (2000) 437-458.

[8] G. Toth and P. Valtr, "The Erdôs-Szekeres theorem: upper bounds and related results", in Combinatorial and computational geometry, Math. Sci. Res. Inst. Publ. 52, (Cambridge Univ. Press, Cambridge, 2005) 557-568.

[9] R. M. Wilson and J. H. van Lint, A Course in Combinatorics (Cambridge Univ. Press, New York, 1992). 